\title{
Multihadron production in nuclear and particle interactions
}

\author{
Edward K. Sarkisyan-Grinbaum ${ }^{1,2}$ and Alexander S. Sakharov ${ }^{3,4}$ \\ ${ }^{1}$ The University of Texas at Arlington, Department of Physics, Arlington, TX 76019, USA \\ ${ }^{2}$ Department of Physics, CERN, CH-1211 Geneva 23, Switzerland \\ ${ }^{3}$ Department of Physics, Wayne State University, Detroit, MI 48202, USA \\ ${ }^{4} \mathrm{TH}$ Division, Department of Physics, CERN, CH-1211 Geneva 23, Switzerland
}

DOI: http://dx.doi.org/10.5689/UA-PROC-2010-09/13

\begin{abstract}
We study the energy-dependence of charged particle mean multiplicity and pseudorapidity density at midrapidity measured in nucleus-nucleus and (anti)proton-proton collisions in the entire available energy range. The study is performed using a model, which considers the multiparticle production process according to the dissipating energy of the participants and their types, namely a combination of the constituent quark picture together with Landau relativistic hydrodynamics. Measurements in nuclear reactions are shown to be well reproduced by the measurements in $\mathrm{pp} / \overline{\mathrm{p}} \mathrm{p}$ interactions and the corresponding fits are presented. Predictions are made for measurements at the forthcoming LHC energies.
\end{abstract}

1. Multiparticle production of soft hadron is one of the most intriguing topics in highenergy interaction studies. Data have been investigated in different types of interactions, ranging from lepton-lepton to nucleus-nucleus (AA) interactions, and over a large energy span, covering several orders of magnitude. QCD, the theory of strong interactions, has provided partonic description of many observations. However, the problem of soft multiparticle production still eludes a complete understanding and remains one of the challenging problems in high-energy physics [1]. The new high-energy data from LHC provide an opportunity to look at the system under new conditions. Of special interest are AA collisions, probing nuclear matter at extreme conditions. The data available from RHIC experiments allow an interesting comparison of the particle production mechanisms with the less complex $\mathrm{e}^{+} \mathrm{e}^{-}$and pp systems. In this context, the global variables such as the average charged particle multiplicity and particle densities (spectra), which are the first available experimental observables, are of fundamental interest $[1,2,3]$ as they are sensitive to the underlying interaction dynamics.

In this report we consider the center-of-mass (c.m.) energy dependence of the average multiplicity and near midrapidity density of charged hadrons produced in AA and pp/p p collisions. Whereas the multiplicity is sensitive mostly to the fraction of energy being transformed into observed particles in a given reaction, the midrapidity density reflects different stages of the reaction. Both variables increase with the collision c.m. energy. Recent measurements at RHIC follow the trends observed in $\mathrm{e}^{+} \mathrm{e}^{-}$and pp interactions. The values of both global variables are found [4] to be similar when comparing the measurements in $\mathrm{e}^{+} \mathrm{e}^{-}$interactions at the c.m. energy of $\sqrt{s_{\mathrm{ee}}}$, and in most central ("head-on") heavy-ion collisions at the nucleon-nucleon c.m.energy $\sqrt{s_{\mathrm{NN}}}=\sqrt{s_{\text {ee }}}$, where the measurements in the latter case are normalized to the number of pairs of participants ("wounded" nucleons [5]). This phenomenon is found to be 
independent of the type of colliding nucleus for $\sqrt{s_{\mathrm{NN}}}$ between $\sim 20 \mathrm{GeV}$ and $200 \mathrm{GeV}$.

Assuming a universal mechanism of hadron production is present in both types of interaction, and that it is driven only by the amount of energy involved into secondary production, one would expect the same value of the observables to be obtained in pp collisions when $\sqrt{s_{\mathrm{pp}}}$ is almost equal to $\sqrt{s_{\mathrm{NN}}}$. However, comparing these data $[6,7]$ to the measurements from RHIC, one finds $[4,8,9,10,11]$ significantly lower values in hadron-hadron collisions. Furthermore, the recent RHIC data from deuteron-gold interactions at $\sqrt{s_{\mathrm{NN}}}=200 \mathrm{GeV}$ unambiguously points to the same values of the mean multiplicity as measured in $\bar{p} p$ collisions $[4,12]$.

To interpret these findings, we have proposed in [13] a phenomenological description based on the energy dissipation by colliding participants into the state formed during the early stage of the collision. Particle production is then driven by the amount of the initial effective energy deposited in this early phase by the relevant types of participants. The experimental observations referred to above have been shown to be well described by this model and further predictions have been made. Recently, the new and higher-energy data have been added and analyzed in our paper [14], as we report here.

2. We consider the whole process of a collision as the expansion and the subsequent break-up into particles from an initial state, in which the total available energy is assumed to be concentrated in a small Lorentz-contracted volume. This approach resembles the Landau phenomenological hydrodynamical description of multiparticle production in relativistic particle collisions [15]. Though the hydrodynamical description does not match ideally the data on multiparticle production in the whole range of pseudorapidity and different particle species, it gives good agreement with the multiplicity measurements in such different reactions as AA, $\mathrm{pp} / \overline{\mathrm{p}} \mathrm{p}, \mathrm{e}^{+} \mathrm{e}^{-}$and $\nu \mathrm{p}$ collisions demonstrating striking predictive power $[4,10,16,17,18,19]$. Recently, the Landau model prediction for the Gaussian pseudorapidity shape due to the longitudinal particle transport has been shown to reproduce well the RHIC data [11] as well as $[4,11]$ the phenomenon known as the "limiting fragmentation" [21]. This indicates that the main assertions of the Landau approach are useful to estimate fractions of the energy dissipated into particles produced in different reactions, particularly in AA collisions [22].

Once the collision of the two Lorentz-contracted particles has resulted in a fully thermalized system, but before expansion, we assume that the production of secondary particles is defined by the fraction of energy of the participants deposited in the volume of thermalized system at the moment of collision. This implies that there is a difference between results of collisions of structureless and composite particles: in composite particle collisions not all the constituents deposit their energy when they form a small Lorentz-contracted volume of the thermalized initial state. Therefore, in nucleon-nucleon collisions the interactions occur between single constituent, or dressed, quarks in accordance with the additive quark picture [23], and the other quarks are considered to be spectators. Thus the energy of the initial thermalized state which is responsible for the number of produced secondary particles is that of the interacting single quark pair i.e., only about $1 / 3$ of the entire nucleon energy is available for particle production in $\mathrm{pp} / \overline{\mathrm{p}} \mathrm{p}$ collisions.

In AA collisions, however, more than one quark per nucleon interacts due to the large size of the nucleus and to the long travel path inside the nucleus. The more central the AA collision is, the more interactions occur and the larger is the energy available for secondary particle production. In central AA collisions, a contribution of constituent quarks rather than participating nucleons seems to determine particle production and their distributions [25]. In the most central collisions, the density of matter is so high (almost saturated) that all three 
constituent quarks from each nucleon may participate nearly simultaneously in the collision, depositing their energy coherently into the thermalized collision volume. In this case, the entire energy of the participating nucleons is available for multiparticle production in head-on AA collisions. Comparing this to $\mathrm{pp} / \overline{\mathrm{p}} \mathrm{p}$ collisions, where only one out of three constituent quarks from each proton interacts, one expects the features of the global variables per pair of participants measured in the most central AA interactions to be similar to those from $\mathrm{pp} / \overline{\mathrm{p} p}$ collisions but at the c.m. energy $\sqrt{s_{\mathrm{pp}}} \simeq 3 \sqrt{s_{\mathrm{NN}}}$.

Combining the above discussed ingredients, one finds for the ratio of the charged particle rapidity density $\rho(y)=\left(2 / N_{\text {part }}\right) d N_{\text {ch }} / d y$ per participant pair at the midrapidity value $y=0$ in AA reaction, $\rho(0)$, to the density $\rho_{\mathrm{pp}}(0)$ in $\mathrm{pp} / \overline{\mathrm{p}} \mathrm{p}$ interaction,

$$
\frac{\rho(0)}{\rho_{\mathrm{pp}}(0)}=\frac{2 N_{\mathrm{ch}}}{N_{\mathrm{part}} N_{\mathrm{ch}}^{\mathrm{pp}}} \sqrt{\frac{L_{\mathrm{pp}}}{L_{\mathrm{NN}}}} .
$$

Here, $N_{\text {part }}$ is the number of participants $\left(N_{\text {part }}=2\right.$ in nucleon-nucleon interactions), $N_{\text {ch }}$ and $N_{\mathrm{ch}}^{\mathrm{pp}}$ are the mean multiplicities in $\mathrm{AA}$ and $\mathrm{pp} / \overline{\mathrm{p} p}$ interactions, respectively, and $L=$ $\ln \frac{\sqrt{s}}{2 m}$ with $m$ being the mass of a participant, e.g. $m=m_{\mathrm{p}}$, the mass of the proton, in AA collisions. According to our model, we compute the ratio (1) for the rapidity density $\rho(0)$ and the multiplicity $N_{\mathrm{ch}}$ at $\sqrt{s_{\mathrm{NN}}}$ and the rapidity density $\rho_{\mathrm{pp}}(0)$ and the multiplicity $N_{\mathrm{ch}}^{\mathrm{pp}}$ at $3 \sqrt{s_{\mathrm{NN}}}$. Due to the above, we consider a constituent quark of mass $\frac{1}{3} m_{\mathrm{p}}$ as a participant in $\mathrm{pp} / \overline{\mathrm{p}} \mathrm{p}$ collisions, and a proton as an effectively structureless participant in head-on AA collisions. Then, from Eq. (1) one obtains:

$$
\rho(0)=\rho_{\mathrm{pp}}(0) \frac{2 N_{\mathrm{ch}}}{N_{\mathrm{part}} N_{\mathrm{ch}}^{\mathrm{pp}}} \sqrt{1-\frac{4 \ln 3}{\ln \left(4 m_{\mathrm{p}}^{2} / s_{\mathrm{NN}}\right)}}, \quad \sqrt{s_{\mathrm{NN}}}=\sqrt{s_{\mathrm{pp}}} / 3 .
$$

$\rho(0)$ is thus calculated from the measured values of $\rho_{\mathrm{pp}}(0)$ and the multiplicities measured in both reactions.

Solving Eq. (1) for $N_{\mathrm{ch}}$ at given $\rho(0), \rho_{\mathrm{pp}}(0)$ and $N_{\mathrm{ch}}^{\mathrm{pp}}$ one finds:

$$
\frac{2 N_{\mathrm{ch}}}{N_{\mathrm{part}}}=N_{\mathrm{ch}}^{\mathrm{pp}} \frac{\rho(0)}{\rho_{\mathrm{pp}}(0)} \sqrt{1-\frac{2 \ln 3}{\ln \left(4.5 \sqrt{s_{\mathrm{NN}}} / m_{\mathrm{p}}\right)}} .
$$

3. Let us first consider the available data on the mean multiplicity and midrapidity density measured in nucleus-nucleus collisions. Figure 1 shows the nuclear data on the pseudorapidity density, $\rho(0)$, per participant pair, measured in head-on nucleus-nucleus collisions, as a function of $\sqrt{s_{\mathrm{NN}}}$ in the energy range $\sqrt{s_{\mathrm{NN}}}=2-200 \mathrm{GeV}$. The data clearly satisfies a linear-log relationship. Fitting, using a weighted combination of the data from the RHIC and SPS experiments, we find:

$$
\rho(0)=(-0.327 \pm 0.026)+(0.381 \pm 0.021) \ln \left(s_{\mathrm{NN}}\right) .
$$

Similar observations have been made in [9, 31, 37]. Such a behaviour seems [19] to be well justified by the participant-driven picture of the formation of the rapidity distribution and the $N_{\text {part }}$ scaling. In addition, as the AA data show, the process of multiparticle production is largely characterized by $N_{\text {part }}$, or soft scaling, rather than by the number of binary collisions, $N_{\text {coll }}$, the latter being attributed to hard scaling $[9,30,31]$. Recently, this feature has been 


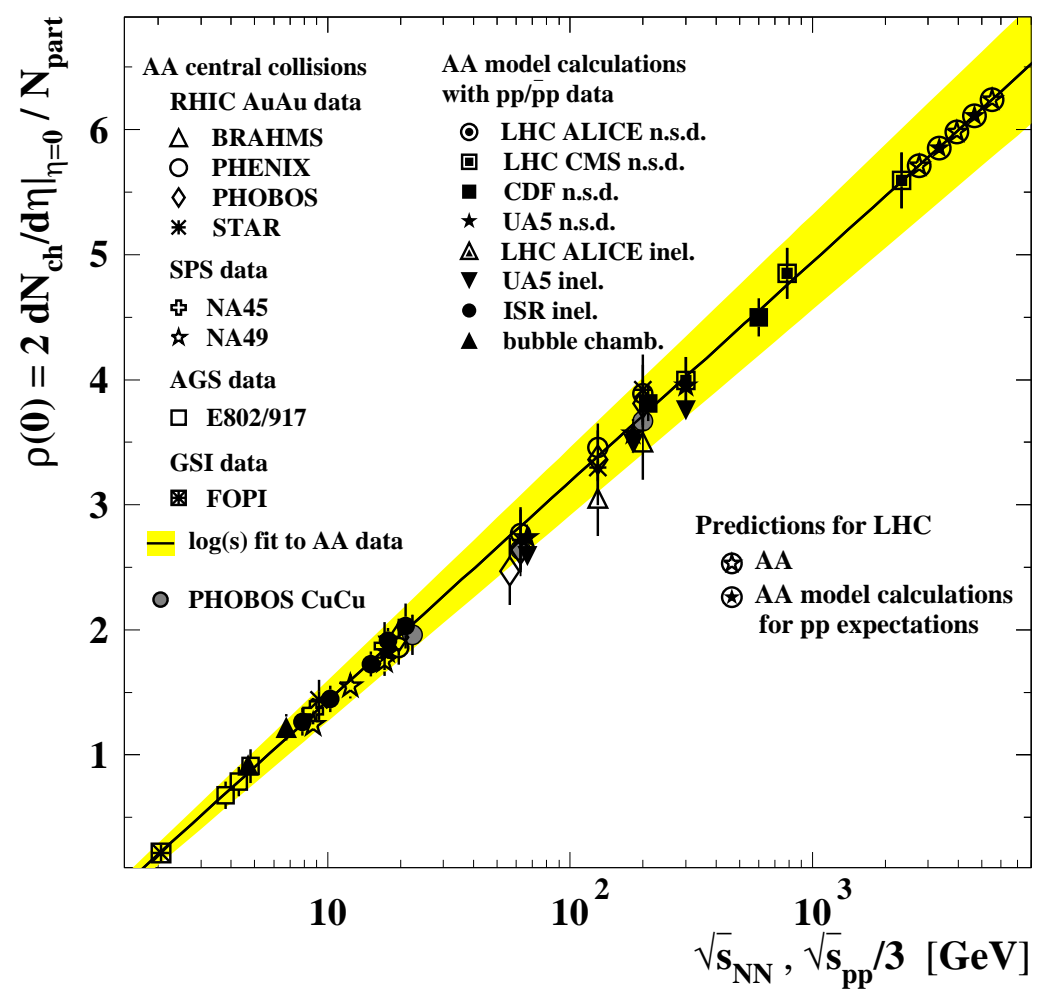

Figure 1: The pseudorapidity density of charged particles per participant pair at midrapidity as a function of c.m. energy per nucleon, $\sqrt{s_{\mathrm{NN}}}$, measured in central nucleus-nucleus (AA) collisions and calculated from pp/pp data using Eq. (2). The AA data are from: the AuAu measurements at RHIC by BRAHMS [11], PHENIX [9], PHOBOS [8, 30, 31, 32, 33], and STAR [34, 35, 36] experiments; the values recalculated in [9] from the measurements at CERN SPS by CERES/NA45 [28] and NA49 [29] experiments, at Fermilab AGS by E802 and E917 experiments [27], and at GSI by FOPI Collab. [26]; the PHOBOS data on $\mathrm{CuCu}$ collisions from [32, 33, 38]. The solid symbols show the values obtained from Eq. (2) using the following data on midrapidity densities measured in non-single diffractive collisions: pp data from ALICE [39] and CMS [40, 41] experiments at LHC and from p p collisions by UA5 Collab. at CERN SPS $[6,42]$ and ISR $\left(\sqrt{s_{\mathrm{pp}}}=53 \mathrm{GeV}\right)$, by CDF Collab. at Fermilab [7]; and in the following inelastic collisions: $\bar{p}$ p data by UA5 Collaboration and pp data from the ALICE [39] experiment and from the ISR [43] and bubble chamber [44, 45] experiments, the latter as recalculated in [6]. The solid line shows the linear-log fit, $-0.33+0.38 \ln \left(s_{\mathrm{NN}}\right)$, to the AA data with the parameters and errors obtained using a combination of the data from the RHIC and SPS experiments. The shaded area shows 1- $\sigma$ error band to the fitted parameters. The circled stars show the heavy-ion predictions for LHC AA collisions (open stars) and from the expected LHC pp collisions (solid stars), both calculated from the fit. 
confirmed by observing the $N_{\text {part }}$ scaling in the ratio of the charged-particle yields measured at $\sqrt{s_{\mathrm{NN}}}=200 \mathrm{GeV}$ and $62.4 \mathrm{GeV}$, as $\left\langle p_{T}\right\rangle$ increases from $0.25 \mathrm{GeV}$ to $\sim 4 \mathrm{GeV}$ [33]. The increase of the ratio at large $\left\langle p_{T}\right\rangle$ is believed to appear because of the harder spectra at higher $\sqrt{s_{\mathrm{NN}}}$, so as to follow $N_{\text {coll }}$ scaling and not to scale with $N_{\text {part }}$. Interestingly, the $N_{\text {part }}$ scaling is observed to be the same for $\mathrm{AuAu}$ and $\mathrm{CuCu}$ interactions.

Figure 2 shows the $\sqrt{s_{\mathrm{NN}}}$-dependence of the AA data of the mean multiplicity $N_{\text {ch }}$ per participant pair from the most central AA collisions in the same $\sqrt{s_{\mathrm{NN}}}$ range as above. The mean multiplicities are seen to increase as a 2nd-order logarithmic polynomial, and our fit gives:

$$
N_{\mathrm{ch}} /\left(0.5 N_{\text {part }}\right)=(-0.35 \pm 0.28)+(0.24 \pm 0.16) \ln \left(s_{\mathrm{NN}}\right)+(0.24 \pm 0.03) \ln ^{2}\left(s_{\mathrm{NN}}\right),
$$

which well reproduces the energy-dependence of the measurements. This trend holds even for relatively low-energies, as demonstrated by the recent data by HADES [48] displayed in Fig. 2. Such an $\ln ^{2}\left(s_{\mathrm{NN}}\right)$ behaviour is expected $[19,20]$ to appear naturally as a combination of the following features: the Landau model Gaussian shape of the pseudorapidity distribution, the logarithmic increase of the midrapidity density with the c.m. energy, and the limiting fragmentation.

4. Here, the midrapidity density and the multiplicity energy dependencies are analyzed in the framework of the model developed, i.e. applying Eqs. (2) and (3) to calculate these variables in $\mathrm{AA}$ interactions based on $\mathrm{pp} / \overline{\mathrm{p}} \mathrm{p}$ data.

Using the $\mathrm{pp} / \overline{\mathrm{p}} \mathrm{p}$ data on $\rho_{\mathrm{pp}}(0)$ from CERN and Fermilab, the data on multiplicities $N_{\mathrm{ch}}^{\mathrm{pp}}$ in $\mathrm{pp} / \overline{\mathrm{p}} \mathrm{p}$ interactions, and $N_{\mathrm{ch}}$ from Fig. 2, we calculate, according to Eq. (2), the $\rho(0)$ at $\sqrt{s_{\mathrm{NN}}}=\sqrt{s_{\mathrm{pp}}} / 3$, shown in Fig. 1 . One can see that the calculated $\rho(0)$ values are in a very good agreement with the measured $\rho(0)$ 's as well as with the obtained log-fit in the whole measured $\sqrt{s_{\mathrm{NN}}}$ range.

The agreement is more intriguing as one adds the hadronic data at c.m. energies higher than the top RHIC energy, namely the densities for $\bar{p}$ p interactions at $\sqrt{s_{\mathrm{pp}}}=1.8 \mathrm{TeV}$ from Tevatron [7] and $\sqrt{s_{\mathrm{pp}}}=2.36 \mathrm{TeV}[39,40]$ and $7 \mathrm{TeV}$ [41] from the LHC. In Fig. 1 we compare the calculations using Eq. (2) on these TeV-energy data with our linear-log fit. One sees the AA $\rho(0)$ 's from $\sqrt{s_{\mathrm{NN}}}=600$ to $\sim 2.33 \mathrm{TeV}$, calculated from the highest energy hadron data available from Tevatron and LHC, agree well with the AA-fit. This observation justifies the above conclusion from the lower-energy midrapidity density dependence on the types of participants. Note that Eq. (2) shows the need of the Landau hydrodynamical energydissipation $L$-factor to correctly estimate the midrapidity density.

Now, from Eq. (3), we calculate the mean multiplicity $N_{\mathrm{ch}} /\left(0.5 N_{\text {part }}\right)$ for AA interactions from the pp $/ \overline{\mathrm{p}} \mathrm{p}$ measurements of $\rho_{\mathrm{pp}}(0)$ and $N_{\mathrm{ch}}^{\mathrm{pp}},{ }^{1}$ and the corresponding $\rho(0)$ data from Fig. 1. The calculated $N_{\mathrm{ch}} /\left(0.5 N_{\text {part }}\right)$ values are shown in Fig. 2 along with the available AA data. One can see that the calculated $N_{\mathrm{ch}} /\left(0.5 N_{\text {part }}\right)$ values reproduce well the $\log ^{2}\left(s_{\mathrm{NN}}\right)$ fit obtained here and follow the nuclear data points for $\sqrt{s_{\mathrm{NN}}}=2 \mathrm{GeV}$ to about $200 \mathrm{GeV}$.

The calculations for the new higher energy data namely those for $\sqrt{s_{\mathrm{NN}}} \gtrsim 200 \mathrm{GeV}$, are again of a special interest. These calculations help to clarify that for $\sqrt{s_{\mathrm{NN}}} \leqslant 200 \mathrm{GeV}$ it is quite difficult to distinguish between the two fits: the power-law fit $\propto s_{\mathrm{NN}}^{\gamma}$, while, after inclusion of the new higher energy data, the $\log ^{2}\left(s_{\mathrm{NN}}\right)$ function seems to be more preferable.

5. Given the universality of the multiparticle production process over almost three orders of magnitude of $\sqrt{s_{\mathrm{NN}}}$, predictions for the LHC energies can be made.

\footnotetext{
${ }^{1}$ For the ALICE, CMS and CDF multiplicities the E735 power-law fit $N_{\mathrm{ch}}^{\mathrm{pp}}=3.102 s_{\mathrm{pp}}^{0.178}[50]$ is used.
} 


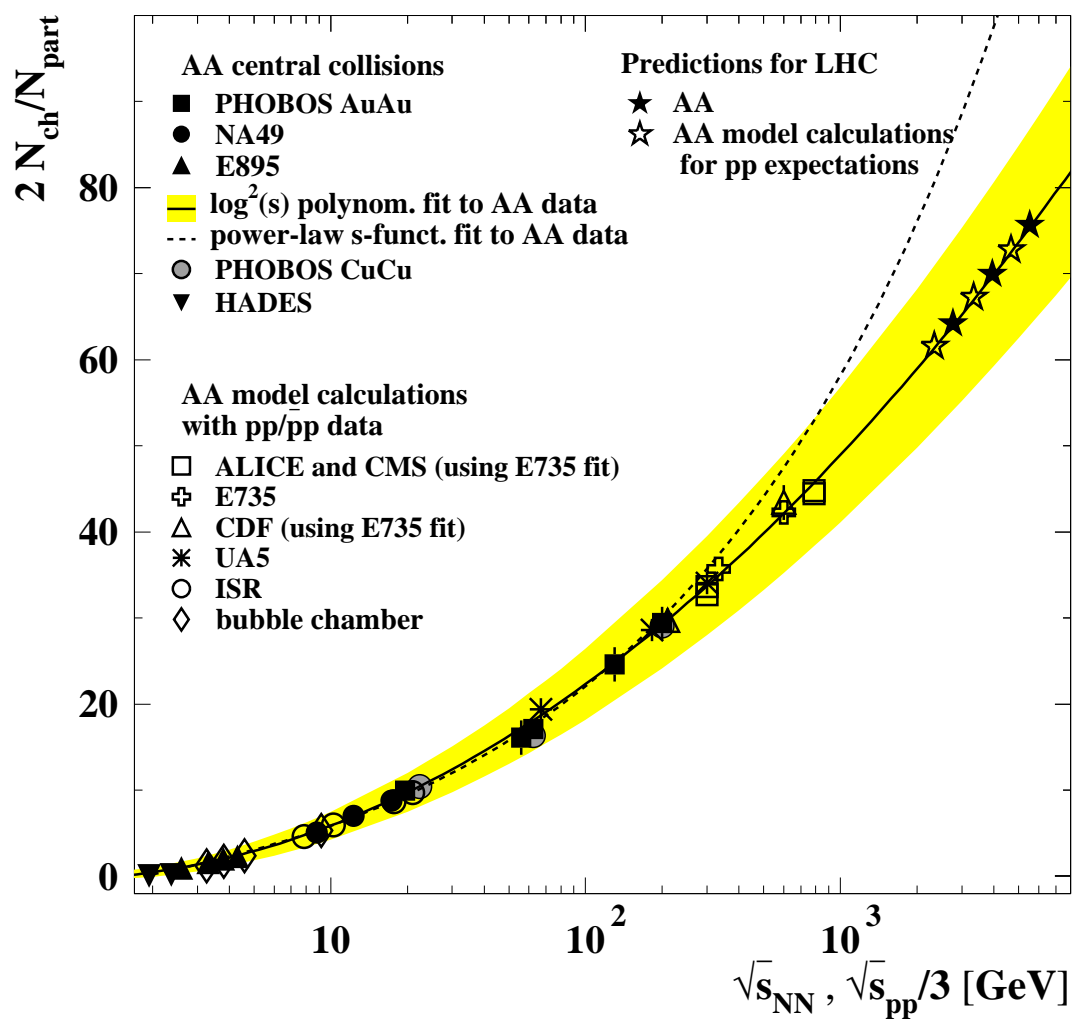

Figure 2: The charged particle mean multiplicity per participant pair as a function of the nucleonnucleon c.m. energy, $\sqrt{s_{\mathrm{NN}}}$, measured in the most central nucleus-nucleus (AA) collisions and calculated using $\mathrm{pp} / \overline{\mathrm{p}} \mathrm{p}$ data from Eq. (3). The solid symbols show the multiplicity values from the AA data as measured by PHOBOS Collab. at RHIC in $\mathrm{AuAu}$ [4, 30, 31] and $\mathrm{CuCu}$ [32] collisions, by NA49 Collab. at CERN SPS [46] and by E895 Collab. at AGS [47] (see also [4]). The low-energy HADES measurements at GSI are taken from [48]. The open symbols show the values obtained from Eq. (3) for the AA mean multiplicity based on: $\bar{p}$ p collisions at FNAL by E735 Collab. [49, 50], at CERN by UA5 Collab. at $\sqrt{s_{\mathrm{pp}}}=546 \mathrm{GeV}$ [42] and $\sqrt{s_{\mathrm{pp}}}=200$ and $900 \mathrm{GeV}$ [51]; pp collisions at CERN-ISR [43], and the ALICE, CMS and CDF multiplicities, calculated using the E735 fit, $3.102 s_{\mathrm{pp}}^{0.178}$ [50], and the data from bubble chamber experiments [45, 52], the latter having been compiled and analysed in [53]. The solid line shows the 2 nd-order $\log$-polynomial fit $-0.35+0.24 \ln \left(s_{\mathrm{NN}}\right)+0.24 \ln ^{2}\left(s_{\mathrm{NN}}\right)$ to the AA data. The shaded area shows 1- $\sigma$ error band to the fitted parameters. The dashed line shows the power-law fit: $-7.32+5.92 s_{\mathrm{NN}}^{0.174}$. The stars give the heavy-ion predictions for the LHC AA collisions (solid symbols) and those expected from LHC pp collisions (open symbols) based on the 2nd-order log-polynomial fit. 
Using the fits, shown in Figs. 1 and 2, and solving Eq. (1) for the midrapidity density $\rho_{\text {pp }}(0)$ with $N_{\mathrm{ch}}^{\mathrm{pp}}$ from the high-energy fit [50], the expected $\rho_{\mathrm{pp}}(0)$ values for pp collisions at LHC are found to be about $5.8,{ }^{2} 6.4$, and 6.9 at $\sqrt{s_{\mathrm{pp}}}=7,10$ and $14 \mathrm{TeV}$, respectively, within $5 \%$ to $10 \%$ uncertainties. From the fit to the midrapidity densities $\rho(0)$, shown in Fig. 1, the $\rho(0)$ values, expected for $\mathrm{PbPb}$ collisions at LHC energies $\sqrt{s_{\mathrm{NN}}}$, corresponding to the above $\sqrt{s_{\mathrm{pp}}}$, are found to be about 5.7, 6.0, and 6.2 at $\sqrt{s_{\mathrm{NN}}}=2.76,3.94$ and $5.52 \mathrm{TeV}$, respectively. The $\rho(0)$ predictions are shown in Fig. 1 by circled solid stars for $\mathrm{PbPb}$ interactions and by circled open stars for those from the pp expectations at LHC at $\sqrt{s_{\mathrm{NN}}}=\sqrt{s_{\mathrm{pp}}} / 3$ when calculated according to our model. These predictions are consistent with other existed expectations [54].

Using the $\log ^{2} s_{\mathrm{NN}}$ fit to the mean multiplicity shown in Fig. 2, one finds the average multiplicity, $N_{\mathrm{ch}} /\left(0.5 N_{\text {part }}\right)$, in $\mathrm{PbPb}$ collisions to be about 64,70 and 73 at $\sqrt{s_{\mathrm{NN}}}=2.76,3.94$ and $5.52 \mathrm{TeV}$, respectively, with $10 \%$ to $15 \%$ uncertainties. The $N_{\mathrm{ch}}^{\mathrm{pp}}$ in pp collisions at LHC are expected to be about 73,82 and 93 at $\sqrt{s_{\mathrm{pp}}}=7,10$ and $14 \mathrm{TeV}$ within about $10 \%$ uncertainties, and are the same as one finds from the multiplicity high-energy power-law fit [50]. The $N_{\text {ch }} /\left(0.5 N_{\text {part }}\right)$ predictions for $\mathrm{PbPb}$ collisions are shown in Fig. 2 by solid stars, while those expected from the LHC pp collisions at $\sqrt{s_{\mathrm{NN}}}=\sqrt{s_{\mathrm{pp}}} / 3$ in the framework of our model, are shown by open stars there.

6. Now, let us dwell on a couple of corollaries of the model proposed here, while more discussion can be found in [14].

From our consideration it follows that, at the same $\sqrt{s_{\mathrm{NN}}}$, the mean multiplicities as well as the midrapidity densities, normalized to the number of participants, would give the similar values when measured in central symmetric AA collisions of different colliding nuclei, they are largely driven by the initial energy deposited by the participants at early stage of collisions. Indeed, as seen from Figs. 1 and 2, this effect has been already observed at SPS energies and now is confirmed by the RHIC measurements at $\sqrt{s_{\mathrm{NN}}}$ of about $50 \mathrm{GeV}$ to $200 \mathrm{GeV}$. The same values for both the observables are obtained [19, 37, 38, 55] in $\mathrm{Au}-\mathrm{Au}$ and $\mathrm{Cu}-\mathrm{Cu}$ data, as shown in Figs. 1 and 2. Note that this effect has also been observed for the whole pseudorapidity region [56].

Considering the above-mentioned similarity in heavy-ion and $\mathrm{e}^{+} \mathrm{e}^{-}$collisions observed, one would expect the same model to be valid in matching the mean multiplicity and the midrapidity values in heavy-ion and $\mathrm{e}^{+} \mathrm{e}^{-}$data. Indeed, as we have shown in [13], both variables follow the same energy dependence within the framework of our picture, as soon as one considers that the structureless electron and positron deposit their total energy into the Lorentz-contracted volume similar to nucleons in central AA collisions. From this, the factor $1 / 3$ applied to the $\mathrm{pp} / \overline{\mathrm{p}} \mathrm{p}$ energy scale is expected to result in a good match between the $\mathrm{e}^{+} \mathrm{e}^{-}$and $\mathrm{pp} / \overline{\mathrm{p}} \mathrm{p}$ data on multiplicity and midrapidity densities as shown in [13]. This solves the problem with the energy-scaling factor of $1 / 2$ used in [4], where the $\sqrt{s_{\mathrm{pp}}} / 2$ shift is shown to provide a reasonable description of the average multiplicity c.m. energy dependence but not of the midrapidity density when comparing heavy-ion $/ \mathrm{e}^{+} \mathrm{e}^{-}$data to those from $\mathrm{pp} / \overline{\mathrm{p}} \mathrm{p}$ collisions. We recall that the energy-scaling factor $1 / 3$, has already been shown in $[57,58]$ to give good agreement of the pp mean multiplicity data relative to those from $\mathrm{e}^{+} \mathrm{e}^{-}$annihilation, for a review see [1]. It is remarkable that the 3NLO perturbative QCD [3] fit to $\mathrm{e}^{+} \mathrm{e}^{-}$data [59] describes the $\mathrm{pp} / \overline{\mathrm{p}} \mathrm{p}$ multiplicity data providing the inelasticity is set to $\approx 0.35$ [50], favouring the effective $1 / 3$ c.m. energy in multihadron production in $\mathrm{pp} / \overline{\mathrm{p}} \mathrm{p}$ reactions.

\footnotetext{
${ }^{2}$ We keep this value as a prediction as soon as it was predicted in our paper [14], on which this report is based, and, as it is seen, the prediction agrees well with the recent CMS measurements [41], shown in Fig. 1
} 
7. In summary, we analyse the average multiplicity and midrapidity density data in $\mathrm{pp} / \overline{\mathrm{p}} \mathrm{p}$ and in central nuclear interactions as a function of the c.m. energy per nucleon over the whole available range of the interaction c.m. energies, including the highest energy LHC data $\sqrt{s_{\mathrm{pp}}}=7 \mathrm{TeV}$ in pp/pp collisions, and the highest energy RHIC data at $\sqrt{s_{\mathrm{NN}}}=200 \mathrm{GeV}$. Within the framework of constituent quarks, we develop a model which interrelates these two variables measured in the two types of interactions, assuming one quark of each nucleon participates in $\mathrm{pp} / \overline{\mathrm{p}} \mathrm{p}$ collision while all three quarks participate in a head-on AA collision. We consider these participants to form the initial zone of a collision which then develops in hydrodynamic framework, the Landau relativistic hydrodynamic model in our case. In this approach soft hadron production is determined at the very early stage of the collision. After appropriately taking into account the contributions of the participants, the average multiplicity and mid-rapidity density in nucleon-nucleon and nucleus-nucleus interactions are found to have a similar c.m. energy dependence. Assuming no changes in the multihadron production processes with increasing energy $\sqrt{s_{\mathrm{pp}}}$ of the LHC and looking forward to the heavy-ion data at the corresponding $\sqrt{s_{\mathrm{NN}}}$, we estimate the multiplicities and midrapidity densities for the forthcoming data, using the obtained energy dependencies.

\section{References}

[1] W. Kittel, E.A. De Wolf, Soft Multihadron Dynamics (World Scientific, 2005), 652 pp.

[2] J. Manjavidze, A. Sissakian, Phys. Rep. 346 (2001) 1;

[3] I.M. Dremin, J.W. Gary, Phys. Rep. 349 (2001) 301.

[4] PHOBOS Collab., B.B. Back et al., Nucl. Phys. A 757 (2005) 28.

[5] A. Białas, B. Bleszyński, W. Czyż, Nucl. Phys. B 111 (1976) 461.

[6] UA5 Collab., G.J. Alner et al., Z. Phys. C 33 (1986) 1.

[7] CDF Collab., F. Abe et al., Phys. Rev. D 41 (1990) 2330.

[8] PHOBOS Collab., B.B. Back et al., Phys. Rev. Lett. 85 (2000) 3100.

[9] PHENIX Collab., S.S. Adler et al., Phys. Rev. C 71 (2005) 034908, 71 (2005) 049901 (E).

[10] PHENIX Collab., K. Adcox et al., Nucl. Phys. A 757 (2005) 184.

[11] BRAHMS Collab., I. Arsene et al., Nucl. Phys. A 757 (2005) 1.

[12] B.B. Back et al., Phys. Rev. C 72 (2005) 031901.

[13] E.K.G. Sarkisyan, A.S. Sakharov, hep-ph/0410324, AIP Conf. Proc. 828 (2005) 35.

[14] E.K.G. Sarkisyan, A.S. Sakharov, arXiv:1004.4390 (2010).

[15] L.D. Landau, Izv. Akad. Nauk: Ser. Fiz. 17 (1953) 51; Englsh translation: Collected Papers of L.D. Landau, Ed. D. Ter-Haarp (Oxford: Pergamon, 1965), p. 569.

[16] E.L. Feinberg, in: Relativistic Heavy Ion Physics: Int. Rev. Nucl. Phys., Vol. 6, Eds. L.P. Csernai, D.D. Strottman (World Scientific, 1991), p. 341, and refs. therein.

[17] P.A. Carruthers, Minh Duong-van, Phys. Rev. D 8 (1973) 859.

[18] P. Steinberg, J. Phys. Conf. Ser. 9 (2005) 280, Nucl. Phys. A 752 (2005) 423c.

[19] W. Busza, J. Phys. G 35 (2008) 044040, Nucl. Phys. A 830 (2009) 35c.

[20] M.-A. Sanchis-Lozano, Int. J. Mod. Phys. A 24 (2009) 4529.

[21] J. Benecke, T.T. Chou, C.N. Yang, E. Yen, Phys. Rev. 188 (1969) 2159. 
[22] J.D. Bjorken, Phys. Rev. D 27 (1983) 140.

[23] For review, see: V.V. Anisovich, N.M. Kobrinsky, J. Nyiri, Yu.M. Shabelsky, Quark Model and High Energy Collisions (World Scientific, Singapore, 2004).

[24] M. Basile et al., Nuovo Cim. A 66 (1981) 129, 73 (1983) 329.

[25] S. Eremin, S. Voloshin, Phys. Rev. C 67 (2003) 064905.

[26] Estimated in [9] from: FOPI Collab., W. Reisdorf et al., Nucl. Phys. A 612 (1997) 493.

[27] E802 Collab., L. Ahle et al., Phys. Rev. C 59 (1999) 2173; E917 Collab., B.B. Back et al., Phys. Rev. Lett. 86 (2001) 1970.

[28] F. Ceretto (for the CERES/NA45 Collab.), Nucl. Phys. A 638 (1998) 467c.

[29] F. Siklér (for the NA49 Collab.) Nucl. Phys. A 661 (1999) 45c.

[30] PHOBOS Collab., B.B. Back et al., Phys. Rev. C 70 (2004) 021902(R).

[31] PHOBOS Collab., B.B. Back et al., Phys. Rev. C 74 (2006) 021901(R).

[32] B. Alver et al., Phys. Rev. Lett. 102 (2009) 142301.

[33] PHOBOs Collab., B. Alver et al., Phys. Rev. C 80 (2009) 011901(R).

[34] STAR Collab., C. Adler et al., Phys. Rev. Lett. 87 (2001) 112303.

[35] STAR Collab., B.I. Abelev et al., Phys. Rev. C 79 (2009) 034909.

[36] STAR Collab., B.I. Abelev et al., Phys. Rev. C 81 (2010) 024911.

[37] R. Nouicer, AIP Conf. Proc. 828 (2005) 11, 842 (2006) 86.

[38] R. Nouicer, arXiv:0901.0910 [nucl-ex].

[39] ALICE Collab., K. Aamondt et al., Eur. Phys. J. C 68 (2010) 89.

[40] CMS Collab., V. Khachatryan et al., JHEP02 (2010) 041.

[41] CMS Collab., V. Khachatryan et al., Phys. Rev. Lett. 105 (2010) 022002.

[42] UA5 Collab., G.J. Alner et al., Phys. Rep. 154 (1987) 247.

[43] W. Thomé et al., Nucl. Phys. B 129 (1977) 365.

[44] J. Whitmore et al., Phys. Rep. 10C (1974) 273.

[45] W.M. Morse et al., Phys. Rev. D 15 (1977) 66.

[46] NA49 Collab., S.V. Afanasiev et al., Phys. Rev. C 66 (2002) 054902.

[47] E895 Collab.: J.L. Klay, PhD Thesis (U.C. Davis, 2001), see [4].

[48] HADES Collab., G. Agakishev et al., Eur. Phys. J. A 40 (2009) 45.

[49] C.S. Lindsey, Nucl. Phys. A 544 (1992) 343c.

[50] J.F. Grosse-Oetringhaus, K. Reygers, J. Phys. G 37 (2010) 083001.

[51] UA5 Collab., R.E. Ansorge et al., Z. Phys. C 43 (1989) 357.

[52] V.Ammosov et al., Phys.Lett. B 42 (1972)519; C.Bromberg et al., Phys. Rev. Lett. 31 (1974) 254.

[53] E. De Wolf, J.J. Dumont, F. Verbeure, Nucl. Phys. B 87 (1975) 325.

[54] N. Armesto: Quark-Gluon Plasma 4, Eds. R.C. Hwa, X.-N. Wang (World Scientific, 2010), 375.

[55] R. Nouicer, Eur. Phys. J. C 49 (2007) 281.

[56] A. Białas, A. Bzdak, Phys. Rev. C 77 (2008) 034908.

[57] P.V. Chliapnikov, V.A. Uvarov, Phys. Lett. B 251 (1990) 192.

[58] T.F. Hoang, Z. Phys. C 62 (1994) 481.

[59] ALEPH Collab., A. Heister et al., Eur. Phys. J. C 35 (2004) 457. 